IJMMS 28:12 (2001) 733-741

PII. S0161171201006639

http://ijmms.hindawi.com

(c) Hindawi Publishing Corp.

\title{
A NEW APPROACH FOR STUDYING FUZZY FUNCTIONAL EQUATIONS
}

\author{
ELIAS DEEBA and ANDRE DE KORVIN
}

(Received 29 January 2001)

\begin{abstract}
We define the concept of a "largest" and a "smallest" solution to an underlying equation and then show that a fuzzy solution of the corresponding fuzzy equation is bounded above and below by these solutions, respectively.
\end{abstract}

2000 Mathematics Subject Classification. 03E72, 39A99.

1. Introduction. The study of functional equations with its wide range of applications is an interesting subject that dates back to the work of Abel. Mathematical models of numerous applied problems result in either ordinary differential, partial differential, difference or algebraic equations. It is standard to assume that the sought variable in these equations is deterministic and can be obtained using numerical or analytical methods. However, in modeling applied problems only partial information may be known or there may be a degree of uncertainty in the parameters used in the model or some measurements may be imprecise. Because of such features, we are tempted to consider the study of functional equations in the fuzzy setting. Indeed, in $[1,2,3,4,5]$ the authors did initiate this study. The methods presented for solving the fuzzy functional equations depended on having a solution to the classical equations for end points of $\alpha$-cuts. In this paper, we present a rather different approach that will provide an upper and a lower bound of the solution. In Section 2, we will discuss the basic background needed for the manuscript and in Section 3, we will present the main results of the paper along with examples.

2. Preliminaries. We present, for the sake of completeness, some background material needed in the sequel. For a detailed study, we refer the reader to $[6,7,8,9,10$, $11,12,13,14,15,16]$. Let $X$ be any nonempty set. The set $A$ is a fuzzy set on $X$ if $A$ is a function from $X$ into the interval [0,1]. The value $A(x)$ is sometimes referred to as the membership of $x$ in $A$.

The set $A$ is said to be convex if every $t \in[0,1], A\left(t x_{1}+(1-t) x_{2}\right) \geq \min \left\{A\left(x_{1}\right)\right.$, $\left.A\left(x_{2}\right)\right\}$. $A$ is said to be normalized if there exists an $x$ such that $A(x)=1$. By the $\alpha$-level of a fuzzy subset $A$, denoted by $[A]_{\alpha}$, we mean

$$
[A]_{\alpha}=\{x \in X \mid A(x) \geq \alpha\} .
$$

It is well known that the $\alpha$-levels of a fuzzy subset $A$ determine $A$. Also, it can be easily verified that for any fuzzy subset $A$ of $X$ :

(C1) $A$ is convex if and only if $[A]_{\alpha}$ is convex and 
(C2) if $A$ is continuous, then $A$ is convex if and only if $[A]_{\alpha}$ is a closed interval. In this setting by a fuzzy number $N$, we mean a fuzzy subset of $X$, the set of positive reals, which is continuous, convex, normalized and vanishing at infinity. Define a function $g: X \rightarrow X$. The extension principle, in one dimension (see $[12,13,14,15])$ states that $g$ can be extended to fuzzy subsets of $X$ as follows:

$$
g(A)(y)=\sup \left(A\left(y_{1}\right)\right)
$$

where the sup is taken over all $y_{1}$ such that $g\left(y_{1}\right)=y$ and $A$ is a fuzzy subset of $X$. Finally, we will need a basic result of Nguyen [10] which addresses the following question. When does the relation

$$
[g(A)]_{\alpha}=g\left([A]_{\alpha}\right)
$$

hold for any continuous function $g$ and for any fuzzy subset $A$ ? It was shown in [10] that (2.3) holds if and only if the sup in (2.2) is attained. That is, there exists $x^{*}$ such that

$$
g(A)(z)=A\left(x^{*}\right)
$$

where $z=g\left(x^{*}\right)$.

3. Main results. Before we discuss the bounds on the fuzzy solution to a functional equation we need the following lemmas. First, we will define the notion of the "largest" solution. We consider the equation

$$
\sup _{t}\{x(t) \wedge A(t)\}=\lambda,
$$

where $A(t)$ is a given function of $t, \lambda$ is a known parameter, $0 \leq \lambda \leq 1$ and $x(t)$ is an unknown function. If there is a solution $x(t), 0 \leq x(t) \leq 1$, then we claim that the "largest solution" to (3.1) is

$$
x_{\infty}^{\prime}(t)= \begin{cases}1, & A(t) \leq \lambda, \\ \lambda, & A(t)>\lambda .\end{cases}
$$

First we show that $x_{\infty}^{\prime}$ is a solution. Indeed,

$$
\begin{array}{ll}
x_{\infty}^{\prime}(t) \wedge A(t) \leq \lambda, & \text { if } A(t) \leq \lambda, \\
x_{\infty}^{\prime}(t) \wedge A(t) \leq \lambda, & \text { if } A(t)>\lambda .
\end{array}
$$

Thus $\sup _{t}\left\{x_{\infty}^{\prime}(t) \wedge A(t)\right\} \leq \lambda$ and $0 \leq x_{\infty}^{\prime}(t) \leq 1$. Moreover, if $A(t)$ exceeds or assumes the value of $\lambda$ for some value $t$, then $\sup _{t}\left\{x_{\infty}^{\prime}(t) \wedge A(t)\right\}=\lambda$. We now show that $x_{\infty}^{\prime}$ is the largest solution. If $x(t)$ is any other solution satisfying (3.1), then for $A(t)>\lambda$, $x(t) \leq \lambda=x_{\infty}^{\prime}(t)$ and for $A(t) \leq \lambda, x(t) \leq 1=x_{\infty}^{\prime}(t)$.

We have, therefore, shown the following lemma.

LEMMA 3.1. The function $x_{\infty}^{\prime}$ is the largest solution to (3.1) among all solutions $x$, $0 \leq x(t) \leq 1$, provided $A(t) \geq \lambda$ for some $t$. 
Next, we define the notion of "smallest" solution. To this end, we consider the equation

$$
\inf _{t}\{x(t) \vee B(t)\}=\mu,
$$

where $B(t)$ is a given function of $t, \mu$ is a known parameter, $0 \leq \mu \leq 1$ and $x(t)$ is an unknown function. If there is a solution $x(t), 0 \leq x(t) \leq 1$, then we claim that the "smallest solution" to (3.4) is

$$
x_{\infty}^{\prime \prime}(t)= \begin{cases}0, & B(t) \geq \mu, \\ \mu, & B(t)<\mu .\end{cases}
$$

We first show that $x_{\infty}^{\prime \prime}(t)$ is a solution. Indeed,

$$
\begin{array}{ll}
x_{\infty}^{\prime \prime}(t) \vee B(t) \geq \mu, & \text { if } B(t) \geq \mu, \\
x_{\infty}^{\prime \prime}(t) \vee B(t) \geq \mu, & \text { if } B(t)<\mu .
\end{array}
$$

Thus $\inf _{t}\left\{x_{\infty}^{\prime \prime}(t) \vee B(t)\right\} \geq \mu$ and $0 \leq x_{\infty}^{\prime \prime}(t) \leq 1$. Moreover, if $B(t)$ is less than or assumes the value of $\mu$ for some value $t$, then $\inf _{t}\left\{x_{\infty}^{\prime \prime}(t) \vee B(t)\right\}=\mu$. We now show that $x_{\infty}^{\prime \prime}$ is the smallest solution. If $x(t)$ is any other solution satisfying (3.4), then for $B(t)<\mu, \mu=x_{\infty}^{\prime \prime}(t) \leq x(t)$ and for $B(t) \geq \mu, 0=x_{\infty}^{\prime \prime}(t) \leq x(t)$.

Thus we have shown the following lemma.

LEMMA 3.2. The function $x_{\infty}^{\prime \prime}$ is the smallest solution of (3.4) among all solutions $x(t)$, $0 \leq x(t) \leq 1$, provided $B(t) \leq \mu$ for some $t$.

REMARK 3.3. The computation of $\lambda$ and $\mu$ for a specified choice of $A(t)$ and $B(t)$ is central for determining the bounds on the solution. We now look at the equation

$$
x_{n+1}=f\left(x_{n}\right)
$$

with given initial condition $x_{0}$. We assume $x_{0}$ is a fuzzy subset of $R$ and we extend $f$ to a fuzzy subsets of $R$ using the extension principle. Then the difference equation reads

$$
x_{n+1}(t)=f\left(x_{n}\right)(t)=\sup \left(x_{n}\left(t_{1}\right)\right),
$$

where the sup is taken over all $t_{1}$ for which $f\left(t_{1}\right)=t$. However, for simplicity of notation we write (3.8) as

$$
x_{n+1}(t)=f\left(x_{n}\right)(t)
$$

We assume that

(1) $f: \mathbb{R} \rightarrow \mathbb{R}$ is a one-to-one function. Thus $f^{-n}$ is also a one-to-one function for every $n\left(f^{-1}\right.$ denotes the inverse of $f$ ),

(2) $\lim _{n \rightarrow \infty} f^{-n}(t)$ is either $\varnothing$ or a singleton for all $t$. If it is a point, we denote this by $f^{-\infty}(t)$.

We may define the fuzzy solution as

$$
x_{\infty}(t)=x_{0}\left[f^{-\infty}(t)\right]
$$


The motivation for this definition comes from the extension principle where

$$
x_{n}(t)=\sup _{\vee \in f^{-n}(t)} x_{0}(\vee)
$$

and the assumption that $f$ is one-to-one $x_{n}(t)=x_{0}\left(f^{-n}(t)\right)$. Then as $n \rightarrow \infty$, we get the above definition.

The problem we consider is as follows: if we put certain bounds on the initial fuzzy set $x_{0}$, what bounds do we have on the fuzzy solution $x_{\infty}$ ?

THEOREM 3.4. Let $f: \mathbb{R} \rightarrow \mathbb{R}$ be a one-to-one function such that $\lim _{n \rightarrow \infty} f^{-n}(t)=$ $f^{-\infty}(t)$ for all $t$. Assume there exist functions $A(t)$ and $B(t)$ with values in $[0,1]$ such that

$$
\begin{gathered}
\sup _{t}\left\{x_{0}\left(f^{-\infty}(t)\right) \wedge A(t)\right\}=\lambda, \\
\inf _{t}\left\{x_{0}\left(f^{-\infty}(t)\right) \vee B(t)\right\}=\mu,
\end{gathered}
$$

and $A(t) \geq \lambda$ and $B(t) \leq \mu$ for some $t$. Then the largest solution, $x_{\infty}^{\prime}$, and smallest solution, $x_{\infty}^{\prime \prime}$, are respectively the upper and lower bounds of the solution to (3.9) with fuzzy initial condition $x_{0}$.

Proof. The equation $x_{n+1}=f\left(x_{n}\right)$ with initial condition $x_{0}$ has formally the solution $x_{n+1}=f^{n+1}\left(x_{0}\right)$. The fuzzy extension of this is

$$
x_{n+1}(t)=\sup _{\vee \in f^{-(n+1)}(t)} x_{0}(\vee)
$$

Since $f^{-1}$ is one-to-one and since $f^{-\infty}(t)=\lim _{n \rightarrow \infty} f^{-(n+1)}(t)$ exists, we can write the above expression as

$$
x_{n+1}(t)=x_{0}\left(f^{-(n+1)}(t)\right)
$$

or write the solution as

$$
x_{\infty}(t)=x_{0}\left(f^{-\infty}(t)\right)
$$

Thus

$$
\sup _{t}\left\{x_{\infty}(t) \wedge A(t)\right\}=\lambda, \quad \inf _{t}\left\{x_{\infty}(t) \vee B(t)\right\}=\mu .
$$

The proof then follows from Lemmas 3.1 and 3.2.

EXAMPLE 3.5. Consider the standard equation

$$
x_{n+1}=\alpha x_{n}
$$

with specified initial condition $x_{0}$ and known $\alpha$. The solution to this equation is given by

$$
x_{\infty}=\lim _{n \rightarrow \infty} \alpha^{n} x_{0}= \begin{cases}0, & \alpha<1, \\ x_{0}, & \alpha=1, \\ \infty, & \alpha>1 .\end{cases}
$$

In this example $f(t)=\alpha t$. We consider each case separately. 
CASE 1. Consider that $\alpha<1$. In this case

$$
f^{-\infty}(t)= \begin{cases}\infty, & t \neq 0 \\ 0, & t=0\end{cases}
$$

Assume that $A(t)=\chi_{a}(t)$, the characteristic function for a given $a \in \mathbb{R}$. We will look for the largest

$$
\lambda=\sup _{t}\left\{x_{0}\left(f^{-\infty}(t)\right) \wedge A(t)\right\}=\chi_{x_{0}}(0) \wedge \chi_{a}(0) .
$$

If $x_{0} \neq 0$ or $a \neq 0$, then $\lambda=0$. In this case

$$
x_{\infty}^{\prime}(t)= \begin{cases}1, & t \neq a, \\ 0, & t=a .\end{cases}
$$

If $x_{0}=a=0$, then $\lambda=1$ and $x_{\infty}^{\prime}(t)=1$ for all $t$. In either case, $x_{\infty} \leq x_{\infty}^{\prime}$.

CASE 2. Consider $\alpha=1$. Note that $f^{-1}(t)=t$. In this case,

$$
\lambda=\sup _{t}\left\{x_{x_{0}}(t) \wedge x_{a}(t)\right\}= \begin{cases}0, & x_{0} \neq a, \\ 1, & x_{0}=a .\end{cases}
$$

Thus when $x_{0} \neq a, x_{\infty}^{\prime}(t)=1$ and $x_{\infty}^{\prime}\left(x_{0}\right)=1$; when $x_{0}=a, x_{\infty}^{\prime}(t)=1$ for all $t$. In either case, $x_{\infty} \leq x_{\infty}^{\prime}$.

CASE 3. Consider $\alpha>1$. In this case, $x_{\infty}=\infty$ means that no $t$ is a solution to the given equation. In this case,

$$
\begin{gathered}
\lambda=\sup _{t}\left\{x_{x_{0}}(0) \wedge x_{a}(t)\right\}=0, \quad x_{0} \neq 0, \\
x_{\infty}^{\prime}(t)= \begin{cases}1, & x \neq a, \\
0, & x=a .\end{cases}
\end{gathered}
$$

Again, in this case $x_{\infty} \leq x_{\infty}^{\prime}$.

We now look for the smallest solution. When $\alpha<1$ and $x_{0} \neq 0$

$$
\mu=\inf _{t}\left\{x_{x_{0}}(0) \vee x_{b}(t)\right\}
$$

If $x_{0} \neq 0$ and we pick $t \neq b, \mu=0$. Thus $x_{\infty}^{\prime \prime}(t)=0$ for all $t$. If $x_{0} \neq 0$, we have when $\alpha=1$ or $\alpha \neq 1, \mu=0$. Thus, $x_{\infty}^{\prime \prime}(t)=0$ for all $t$. Hence $x_{\infty}^{\prime \prime} \leq x_{\infty}$.

The previous techniques may be applied to solutions where much less information is given on $x_{0}$ and $f$. The next example illustrates this situation.

EXAMPLE 3.6. Assume that $f$ is an increasing function on $[0,1]$ with $f^{\prime \prime}>0$ on $\left[0, t^{*}\right]$ and $f^{\prime \prime}<0$ on $\left[t^{*}, 1\right]$ where $t^{*} \in[0,1]$ is such that $f\left(t^{*}\right)=t^{*}$. Then $f^{-\infty}(t)=t^{*}$ for $t \neq 0, t \neq 1$ and $f^{-\infty}(0)=0$ and $f^{-\infty}(1)=1$. Assume that $x_{0}, A$, and $B$ are fuzzy subsets of $[0,1]$. Then

$$
\begin{aligned}
& \lambda=\left[x_{0}\left(t^{*}\right) \wedge A\left(t^{*}\right)\right] \vee\left[x_{0}(0) \wedge A(0)\right] \vee\left[x_{0}(1) \wedge A(1)\right], \\
& \mu=\left[x_{0}\left(t^{*}\right) \vee B\left(t^{*}\right)\right] \wedge\left[x_{0}(0) \vee B(0)\right] \wedge\left[x_{0}(1) \vee B(1)\right]
\end{aligned}
$$

and the previous bounds $x_{\infty}^{\prime}$ and $x_{\infty}^{\prime \prime}$ are readily computed. 
In the previous examples $f$ was assumed to be one-to-one function, what happens when $f$ is not one-to-one? In the computation of $\lambda$ and $\mu, x_{0}\left[f^{-\infty}(t)\right]$ must be replaced by $\sup _{\vee \in f^{-}(t)} x_{0}(\vee)$. The next example illustrated this situation.

EXAMPLE 3.7. Consider the equation

$$
x_{n}=4 x_{n-1}\left(1-x_{n-1}\right)
$$

with fuzzy initial condition $x_{0}$. In this example $f(x)=4 x(1-x)$. Assume, for simplicity sake, that $x_{0}, A$, and $B$ are all fuzzy subsets of $[0,1]$. Then $f^{-}(t)=\{(1-$ $\sqrt{1-t}) / 2,(1+\sqrt{1-t}) / 2\}$.

It is easy to see that $f^{-\infty}(t)=\{0,1 / 2,1\}$ for all $t$. If $\sup _{t} A(t)=1$, then

$$
\begin{aligned}
& \lambda=x_{0}(0) \vee x_{0}\left(\frac{1}{2}\right) \vee x_{0}(1), \\
& \mu=x_{0}(0) \vee x_{0}\left(\frac{1}{2}\right) \vee x_{0}(1) \vee \inf _{t}(B(t))
\end{aligned}
$$

and the bound estimates are readily computed.

Note 1. The solution to (3.9) with initial condition $x_{0}$ may be viewed as an intervalvalued fuzzy set.

The bounds previously obtained involve, $0,1, \lambda$, and $\mu$. Can we get more precise estimates? If we consider $\lambda$ and $\mu$ as given by (3.1) and (3.4), we set

$$
\lambda=\operatorname{poss}[x, A], \quad \mu=\operatorname{nec}\left[x, B^{c}\right],
$$

where poss $[x, A]$ is the largest intersection of $x$ and $A$ and nec $\left[x, B^{c}\right]$ is the weakest implication not $B \Rightarrow x$. It therefore makes sense that we could sharpen the bounds if we had a family of known possibilities and known necessities. Again assume $f$ is oneto-one and $f^{-n}(t) \rightarrow f^{-\infty}(t)$ for all $t$. We define "an implication operator" on $[0,1]$ by

$$
a \phi b= \begin{cases}1, & a \leq b, \\ b, & a>b .\end{cases}
$$

We assume that for each $y \in Y$, poss $\left[x_{0} f^{-\infty}, A_{y}\right]$ is known. We set

$$
p(y)=\operatorname{poss}\left[x_{0} f^{-\infty}, A_{y}\right]
$$

and let $u(x)=\inf _{y}\left\{A_{y}(x) \phi p(y)\right\}$.

THEOREM 3.8. Assume that for every $y \in Y$, there exists $x_{y}$ such that $A_{y}\left(x_{y}\right)>$ $p(y)$. Then $u$ as defined above is an upper bound for the solution of (3.30).

Proof. Consider poss $\left[x_{0} f^{-\infty}, A_{y}\right]=p(y)$ for $y \in Y$. By Theorem 3.4, for each fixed $y$, the largest solution is given by

$$
x_{\infty, y}^{\prime}(t)= \begin{cases}1, & A_{y}(t) \leq p(y), \\ p(y), & A_{y}(t)>p(y) .\end{cases}
$$


Therefore, by definition of the implication operator, $\phi$,

$$
x_{\infty, y}^{\prime}(t)=A_{y}(t) \phi p(y) .
$$

Since this is an upper bound for all $y \in Y$, the sharpest upper bound generated is

$$
u(t)=\inf _{y} x_{\infty, y}^{\prime}(t)=\inf _{y}\left\{A_{y}(t) \phi p(y)\right\}
$$

We now apply a similar approach to establish a lower bound for the solution of the difference equation (3.30) with fuzzy initial condition $x_{0}$. The operator analogous to $\phi$ is defined as

$$
a \beta b= \begin{cases}b, & a<b, \\ 0, & a \geq b .\end{cases}
$$

We assume nec $\left[x_{0} f^{-\infty}, B_{y}^{c}\right]$ is known for a family of $y \in Y$. We set

$$
\begin{gathered}
\operatorname{nec}\left[x_{0} f^{-\infty}, B_{y}^{c}\right]=N(y), \\
L(t)=\sup _{y}\left\{B_{y}(t) \beta N(y)\right\} .
\end{gathered}
$$

THEOREM 3.9. Assume that for every $y \in Y$, there exists $x_{y}$ such that $B_{y}\left(x_{y}\right) \leq$ $N(y)$. Then $L$ as defined in (3.35b) is a lower bound of the solution to (3.35a).

Proof. Let nec $\left[x_{0} f^{-\infty}, B_{y}^{c}\right]=N(y)$. By Theorem 3.8, for each $y$, the smallest solution is given by

$$
x_{\infty, y}^{\prime \prime}(t)= \begin{cases}0, & B_{y}(t) \geq N(y), \\ N(y), & B_{y}(t)<N(y) .\end{cases}
$$

Thus, by definition of $\beta, x_{\infty, y}^{\prime \prime}(t)=B_{y}(t) \beta N(y)$. This is a lower bound for all $y$ so the sharpest lower bound generated is

$$
L(x)=\sup x_{\infty, y}^{\prime \prime}(t)=\sup _{y}\left\{B_{y}(t) \beta N(y)\right\}
$$

The previous result can be applied when $x_{0}$ is not completely determined. This is demonstrated in the next example.

EXAMPLE 3.10. Consider a function $f$ such that we may find a partition $P_{i}$ such that $f^{-\infty}\left(P_{i}\right)=t_{i}, 1 \leq i \leq l$. We also assume that $f^{-n}$ converges to a point for all $t$ which we denote by $f^{-\infty}(t)$. Also assume given two fuzzy sets: $A_{y_{1}}$ and $A_{y_{2}}$. Then

$$
\begin{aligned}
& \sup _{i}\left\{x_{0}\left(t_{i}\right) \wedge A_{y_{1}}(t)\right\}=p_{1}=p\left(y_{1}\right), \\
& \sup _{i}\left\{x_{0}\left(t_{i}\right) \wedge A_{y_{2}}(t)\right\}=p_{2}=p\left(y_{2}\right) .
\end{aligned}
$$


The left-hand sides of the above two equations are poss $\left[x_{\infty}, A_{y_{1}}\right]$ and $\operatorname{poss}\left[x_{\infty}, A_{y_{2}}\right]$ where $x_{\infty}$ is a solution to (3.38) with initial fuzzy condition. Now for $k=1,2$

$$
A_{y_{k}}(t) \phi p_{k}= \begin{cases}1, & A_{y_{k}}(t) \leq p_{k} \\ p_{k}, & A_{y_{k}}(t)>p_{k} .\end{cases}
$$

So if $t \in\left[A_{y_{1}}\right]_{p_{1}^{*}} \wedge\left[A_{y_{2}}\right]_{p_{2}^{*}}\left(p_{1}^{*}>p_{1}, p_{2}^{*}>p_{2}\right)$, then $x_{\infty}(t) \leq p_{1} \wedge p_{2}$. We note that $x_{0}\left(t_{i}\right) \wedge \sup _{t \in P_{t}} A_{k}(t)$ can be reinterpreted as follows: let $\hat{A}_{k, i}$ be the restriction of $A_{k}$ to $P_{i}$, then $x_{0}\left(t_{i}\right) \wedge \sup _{t \in \prod_{i}} A_{y_{k}}(t)=\operatorname{poss}\left[P_{i}, \hat{A}_{k, i}\right] \wedge x_{0}\left(t_{i}\right)$, where the set $P_{i}$ is identified with its characteristic function. The possibility of the solution and $A_{k}$ is then given by

$$
\sup _{1 \leq i \leq l}\left\{x_{0}\left(t_{i}\right) \wedge \operatorname{poss}\left[p_{i}, \hat{A}_{k, i}\right] \wedge x_{0}\left(t_{i}\right)\right\}
$$

not knowing the precise value of $x_{0}\left(t_{i}\right)$ but knowing the above expression enables us to assign an upper bound to the solution. A similar example could be constructed for a lower bound.

The approach we have taken here is to define the initial condition, $x_{0}(t)$, as a function of $t$ with values in $[0,1]$. This indicates the degree to which the number $t$ is the initial condition. The bounds obtained on $x_{0}$ along with the assumptions on $f$ led to a solution to the fuzzy difference equation. The novelty of this approach over the one used in $[1,2,3,4,5]$ is that in this approach we do not assume that the classical equation must have an explicit solution. We rather obtain bounds on the solution. The approach can be applied to a variety of problems.

\section{REFERENCES}

[1] E. Deeba and A. de Korvin, On a fuzzy difference equation, IEEE Transaction on Fuzzy Systems 3 (1995), no. 4, 469-472.

[2] E. Deeba, A. de Korvin, and E. L. Koh, A fuzzy difference equation with an application, J. Differ. Equations Appl. 2 (1996), no. 4, 365-374. MR 99b:39006. Zbl 0882.39002.

[3] _ On a fuzzy logistic difference equation, Differential Equations Dynam. Systems 4 (1996), no. 2, 149-156. MR 99g:39006. Zbl 0873.39002.

[4] E. Deeba, A. de Korvin, and S. Xie, Pexider functional equations - their fuzzy analogs, Int. J. Math. Math. Sci. 19 (1996), no. 3, 529-538. MR 97h:39019. Zbl 0852.39006.

[5]___ Techniques and applications of fuzzy set theory to difference and functional equations and their utilization in modeling diverse systems, Fuzzy Theory Systems: Techniques and Applications, vol. 1-4, Academic Press, California, 1999, pp. 87-110. CMP 1737450.

[6] D. Dubois and H. Prade, Operations on fuzzy numbers, Internat. J. Systems Sci. 9 (1978), no. 6, 613-626. MR 58\#10464. Zbl 0383.94045.

[7] _ Fuzzy Sets and Systems. Theory and Applications, Mathematics in Science and Engineering, vol. 144, Academic Press, New York, 1980. MR 82c:03074. Zbl 0444.94049.

[8] H. M. Hersch and A. Camarazza, A fuzzy-set approach to modifiers and vagueness in natural languages, J. Exp. Psychol. General 105 (1976), 254-276.

[9] O. Kaleva, Fuzzy performance of a coherent system, J. Math. Anal. Appl. 117 (1986), no. 1, 234-246. MR 87g:60074. Zbl 0601.90058.

[10] H. T. Nguyen, A note on the extension principle for fuzzy sets, J. Math. Anal. Appl. 64 (1978), no. 2, 369-380. MR 58\#243. Zbl 0377.04004. 
[11] G. C. Oden, Integration of fuzzy logical information, Journal of Experimental Psychology: Human Perception and Performance 3 (1977), no. 4, 565-575.

[12] L. A. Zadeh, Fuzzy sets, Information and Control 8 (1965), 338-353. MR 36\#2509. Zbl 0139.24606 .

[13] _ The concept of a linguistic variable and its application to approximate reasoning. I, Information Sci. 8 (1975), 199-249. MR 52\#7225a. Zbl 0397.68071.

[14]__ The concept of a linguistic variable and its application to approximate reasoning. II, Information Sci. 8 (1975), 301-357. MR 52\#7225b. Zbl 0404.68074.

[15] - The concept of a linguistic variable and its application to approximate reasoning. III, Information Sci. 9 (1975), no. 1, 43-80. MR 52\#7225c. Zbl 0404.68075.

[16] H. J. Zimmermann, Results of empirical studies in fuzzy set theory, Applied General Systems Research (G. J. Klir, ed.), Plenum Press, New York, 1978, pp. 303-312. Zbl 0403.94040.

Elias DeEba: Department of Computer and Mathematical Sciences, University of HOUSTON-DOWNTOWN, ONE MAIN STREET, HOUSTON, TX 77002, USA

E-mail address: deebae@dt.uh.edu

ANDRE DE KORVIN: DePARTMENT OF COMPUTER AND MATHEMATICAL SCIENCES, UNIVERSITY OF HOUSTON-DOWNTOWN, ONE MAIN STREET, HOUSTON, TX 77002, USA

E-mail address: dekorvin@dt.uh.edu 


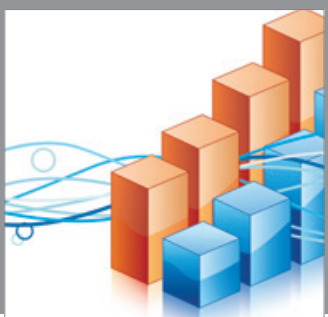

Advances in

Operations Research

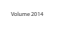

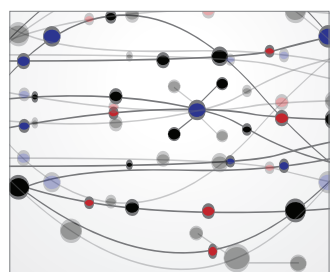

\section{The Scientific} World Journal
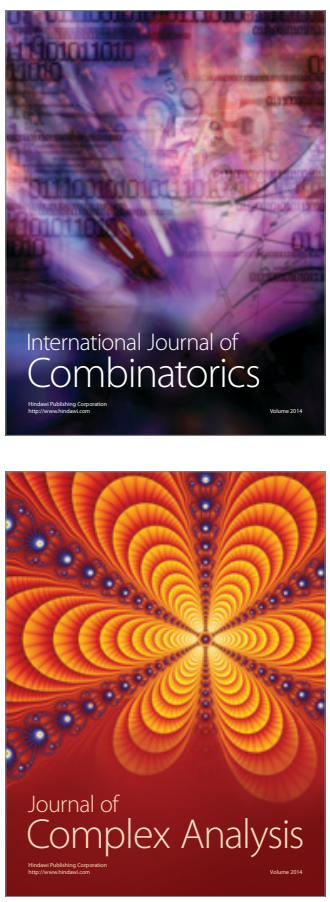

International Journal of

Mathematics and

Mathematical

Sciences
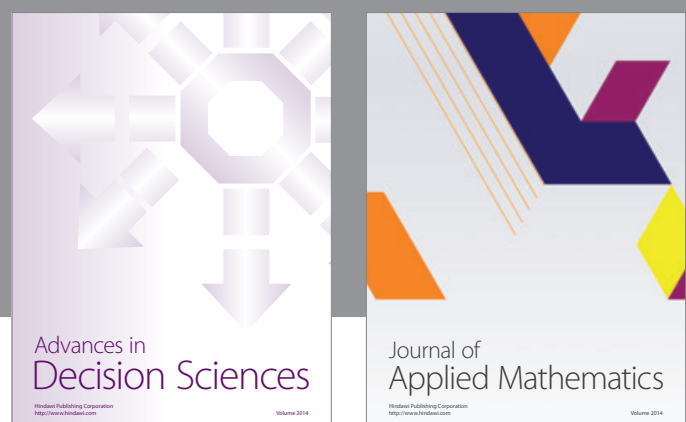

Journal of

Applied Mathematics
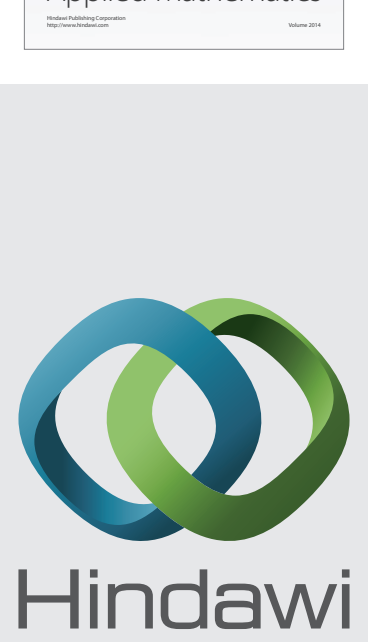

Submit your manuscripts at http://www.hindawi.com
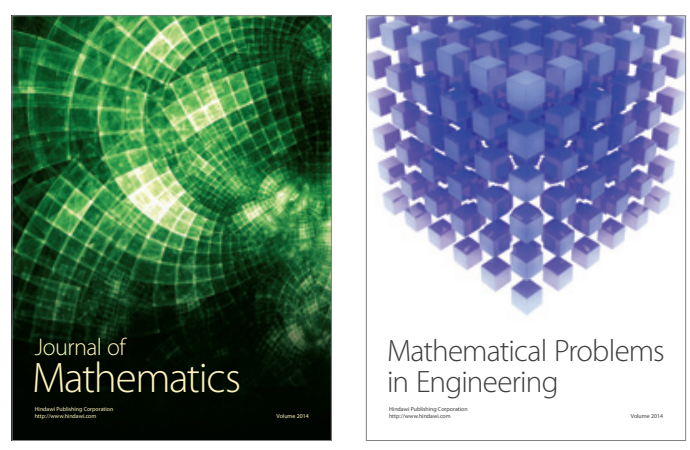

Mathematical Problems in Engineering
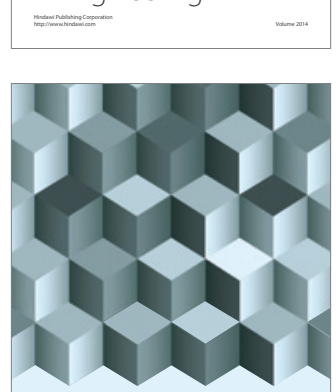

Journal of

Function Spaces
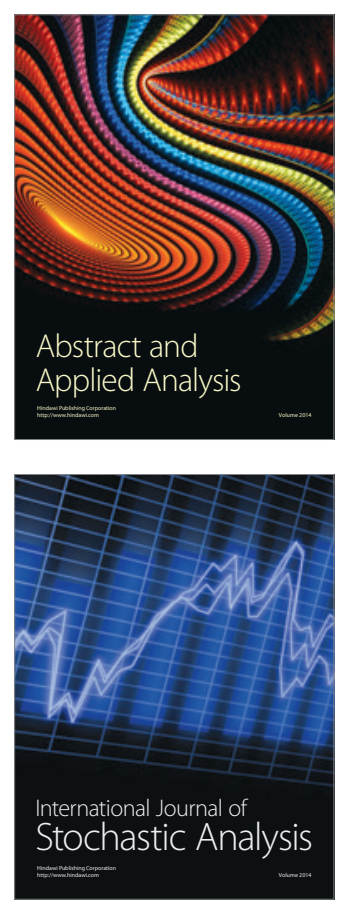

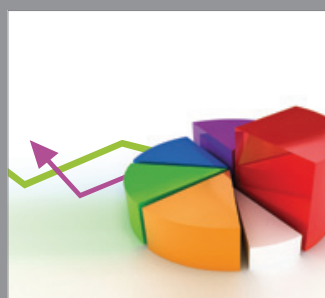

ournal of

Probability and Statistics

Promensencen
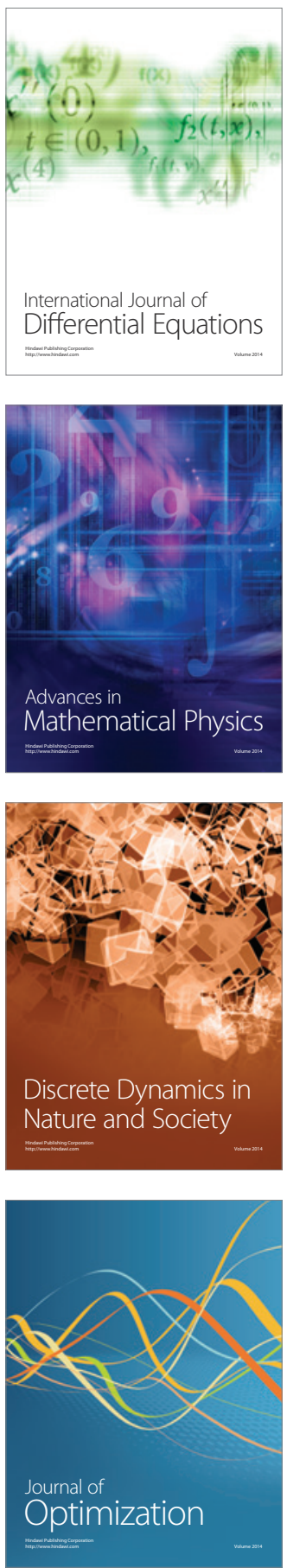